\title{
Pentacyclic Harmonic Graph
}

\author{
Ahmad Salehi Zarrin Ghabaei, Shahroud Azami \\ Department of Mathematics, Parand Branch, Islamic Azad University, Parand, Iran \\ Email address: \\ ahmadpuya@gmail.com (A. S. Z. Ghabaei),azami@sci.ikiu.ac.ir (S. Azami)

\section{To cite this article:} \\ Ahmad Salehi Zarrin Ghabaei, Shahroud Azami. Pentacyclic Harmonic Graph. Pure and Applied Mathematics Journal. \\ Vol. 5, No. 5, 2016, pp. 165-173. doi: 10.11648/j.pamj.20160505.15
}

Received: August 28, 2016; Accepted: September 8, 2016; Published: October 11, 2016

\begin{abstract}
Let $G$ be a graph on n vertices $v_{1}, v_{2}, \ldots, v_{n}$ and let $d\left(v_{i}\right)$ be the degree of vertex $v_{i}$. A graph $G$ is defined to be harmonic if $\left(d\left(v_{1}\right), d\left(v_{2}\right), \ldots, d\left(v_{n}\right)\right)^{t}$ is an eigenvector of the $(0,1)$-adjacency matrix of $G$. We now show that there are 4 regular and 45 non-regular connected pentacyclic harmonic graphs and determine their structure. In the end we conclude that all of c-cyclic harmonic graphs for $1 \leq c \leq 5$ are planar graphs.
\end{abstract}

Keywords: Harmonic Graph, Eigenvalue, Spectra

\section{Introduction}

Let $G=(V(G), E(G))$ be a graph with $|V(G)|=n$ vertices $v_{1}, v_{2}, \ldots, v_{n}$ and $|E(G)|=m$ edges. We say that $G$ is c-cyclic, whenever $c=m-n+p$, which $p$ is the number components of $G$. In [1] B. Borovicanin and et al, studied the c-cycle graphs for $c=1,2,3,4$. All harmonic trees were constructed in [8] and the number of walks counted on some harmonic graph in $[4,5]$. In $[9,10,11]$ founded some result on harmonic graphs.

In this paper we study the c-cycle graphs for $c=5$. If the graph $G$ is connected and $c=0$ then $G$ is a tree.

The following elementary properties of harmonic graphs obtain of the spectra properties of graphs $[2,3,6,7]$. Let $d\left(v_{i}\right)$ be the degree of vertex $v_{i}$ for $1 \leq i \leq n$, that is the number of the first neighbors of $v_{i}$. Vertex of degree $\mathrm{k}$ is called a k-vertex. Vertex of degree zero is called pendent. The column-vector $\left(d\left(v_{1}\right), d\left(v_{2}\right), \ldots, d\left(v_{n}\right)\right)^{t}$ is denoted by $d(G)$. The number of k-vertex denoted by $n_{k}$ and we have

$$
\begin{aligned}
& n_{5}=1 \\
& n_{3}=0
\end{aligned}
$$

Definition 1. The adjacency matrix $A(G)=\left[a_{i j}\right]$ is the $n \times n$ matrix for which $a_{i j}=1$ if $v_{i} v_{j} \in E(G)$ and $a_{i j}=0$ otherwise. Eigenvalues and eigenvectors of matrix $A(G)$ is called eigenvalues and eigenvectors of graph $G$.

Definition 2. A graph $G$ is said to be harmonic if there exists a constant $\lambda$, such that

$$
n_{1}=3, n_{2}=0, n_{4}=3
$$

In other words

$$
A(G) d(G)=\lambda d(G)
$$

Thus, graph $G$ is harmonic if and only if $d(G)$ is one of its eigenvectors, theses graphs are called $\lambda$-harmonic. Equation (3) result that $\lambda$ is a rational number and equation (4) implies that $\lambda$ is not proper fraction, they follows that $\lambda$ must be an integer.

Example 1. A $\lambda$-regular graph is a $\lambda$-harmonic graph.

By summing the expressions (3) over all $i=1,2, \ldots, n$ we have

$$
\sum_{v \in V(G)} d(v)(d(v)-\lambda)=0
$$

equivalently

$$
\sum_{k \geq 0} k(k-\lambda) n_{k}=0
$$




\section{Some Auxiliary Results}

We have the follow results of [1].

\section{Lemma 1.}

i. Let $\mathrm{H}$ be a graph obtained from $\mathrm{G}$ by adding to it an arbitrary number of isolated vertices, then $\mathrm{H}$ is harmonic if and only if $\mathrm{G}$ is harmonic.

ii. Any graph without isolated vertices is $\lambda$-harmonic if and only if all its components are $\lambda$-harmonic.

iii. Let $\mathrm{G}$ be a connected $\lambda$-harmonic graph. Then $\lambda$ is greatest eigenvalue of $\mathrm{G}$ and its multiplicity is one. Also if $m>0$ then $\lambda \geq 1$ and equality occurs if and only if $G=K_{2}$.

From Lemma 2.1., it is enough to restrict our considerations to connected non-regular graphs. In [8], shown that for any positive integer $\lambda$ there is a unique connected $\lambda$-harmonic that is a tree and denoted by $T_{\lambda}$. $T_{\lambda}$ has $\lambda^{3}-\lambda^{2}+\lambda+1$ vertices, of which one vertices is a $\left(\lambda^{2}-\lambda+1\right)$-vertex, $\left(\lambda^{2}-\lambda+1\right)$ vertices are $\lambda$-vertices and $(\lambda-1)\left(\lambda^{2}-\lambda+1\right)$ vertices are pendant. Also in [1], shown that the following lemmas:

\section{Lemma 2.}

a In a $\lambda$-harmonic graph 1 -vertex is adjacent to a vertex of degree $\lambda$.

b If a $\lambda$-harmonic graph not regular, then it has a vertex of degree greater than $\lambda$.

c In a harmonic graph with $n>2$, no 1 -vertex is attached to any vertex of greatest degree.

\section{Lemma 3.}

The tree $T_{2}$ is the unique connected non-regular 2harmonic graph.

\section{Lemma 4.}

If $x$ is a vertex of a $\lambda$-harmonic graph then $d(x) \leq \lambda^{2}-\lambda+1$. If $d(x)=\lambda^{2}-\lambda+1$ then $x$ belongs to a tree $T_{\lambda}$, otherwise $d(x)<\lambda^{2}-\lambda+1$.

Lemma 5: For the $\lambda$-harmonic tree, $n_{1}=(\lambda-1) n_{\lambda}$. For any other connected $\lambda$-harmonic graph, $n_{1} \leq(\lambda-2) n_{\lambda}$.

Lemma 6.

If $G \neq T_{\lambda}$ be a connected c-cyclic $\lambda$-harmonic graph with $\lambda \geq 3$, then $c \geq \frac{1}{2}\left(\lambda^{2}-2 \lambda+2\right)$.

\section{Lemma 7.}

Let $v$ be a vertex of a $\lambda$-harmonic graph such that $d(v)>\lambda^{2}-3 \lambda+4$, and let $u$ be a vertex adjacent to $v$, then $d(u)=\lambda$.

After then, we suppose that c-cyclic graphs are connected, that is $p=1$ therefore $m=n+c-1$. By combining the equalities (1) and (2) we get

$$
\sum_{k \geq 0} k(k-2) n_{k}=2 c-2
$$

\section{The Main Result}

\section{Theorem 1.}

There are exactly 45 non-regular connected pentacyclic harmonic graphs, depicted in Figures 1- 17.

Proof:

Because of Lemma 6 , if $c=5$ then $\lambda$ cannot be greater than 4. Since $c=5$, therefore, $m=n+4$, on the other hand, Lemmas 2 and 5, result that $\lambda$ cannot equal to 1 and 2, hence $\lambda=3$ or $\lambda=4$. At the first, suppose that $\lambda=3$.

By the Lemma 2.4 if $\Delta$ is the maximal degree in a pentacyclic harmonic graph, then $\Delta \leq 6$ and in case $\lambda=4$ by Lemma 4 we have $\Delta \leq 12$. From Lemma 2 we the conclude that only the following 11 cases need to be examined:

$\begin{array}{ll}\text { Case 1: } \lambda=3, & \Delta=6 \\ \text { Case 2: } \lambda=3, & \Delta=5 \\ \text { Case 3: } \lambda=3, & \Delta=4 \\ \text { Case 4: } \lambda=4, & \Delta=12 \\ \text { Case 5: } \lambda=4, & \Delta=11 \\ \text { Case 6: } \lambda=4, & \Delta=10 \\ \text { Case 7: } \lambda=4, & \Delta=9 \\ \text { Case 8: } \lambda=4, & \Delta=8 \\ \text { Case 9: } \lambda=4, & \Delta=7 \\ \text { Case 10: } \lambda=4, & \Delta=6 \\ \text { Case 11: } \lambda=4, & \Delta=5\end{array}$

Case 1: Lemma 5 implies that $n_{3}-n_{1} \geq 0$. By means of relation (7), for $c=5$, we have

$$
-n_{1}+n_{3}+2 n_{4}+3 n_{5}+4 n_{6}=8
$$

from which

$$
2 n_{4}+3 n_{5}+4 n_{6}-8=n_{1}-n_{3} \leq 0
$$

and we can conclude that

$$
1 \leq n_{6} \leq 2, n_{5} \leq 1, n_{4} \leq 2
$$

from equation (1.6) we get

$$
-2 n_{1}-2 n_{2}+4 n_{4}+10 n_{5}+18 n_{6}=0 .
$$

According to Lemma 7, the 5 and 6-vertices are adjacent only to 3 -vertices. Since (3) the two neighbors of every 3vertex, adjacent to a 6-vertex, must be a 1 and 2-vertex. Therefore $n_{1} \geq 6, n_{2} \geq 3$, and consequently, $n_{1}+n_{2} \geq 9$. In what follows we distinguish between 12 subcases:

Subcase 1:

$$
n_{4}=0, n_{5}=0, n_{6}=1, n_{1}+n_{2}=9, n_{3}=n_{1}+4
$$

In this subcase, we have, $n_{1}=6, n_{2}=3, n_{3}=10$, $n_{4}=0, n_{5}=0, n_{6}=1$. Each of the three 2-vertices must be adjacent to two 3-vertices, and exacts of 4, 3-vertices remains. Therefore there cannot exist a 3-harmonic satisfies the condition (12). 


\section{Subcase 2:}

$$
n_{4}=1, n_{5}=0, n_{6}=1, n_{1}+n_{2}=11, n_{3}=n_{1}+2
$$

The 4 and 6-vertices are adjacent only to 3-vertices and therefore the number of 3 -vertices is greater than or equal to 10. Because of $n_{2} \geq 3$ we now have $n_{1}=n_{3}-2 \geq 8$. Then, in this subcase we get

$$
n_{1}=8, n_{2}=3, n_{3}=10, n_{4}=1, n_{5}=0, n_{6}=1
$$

the neighbors 3-vertex adjacent to 4-vertex has a 2-vertex, a 3 -vertex, so we need at least 5, 2-vertices. This subcase also impossible.

Subcase 3:

$$
n_{4}=2, n_{5}=0, n_{6}=1, n_{1}+n_{2}=13, n_{3}=n_{1}
$$

Similar arguments subcase 2, we have

$$
n_{1}=10, n_{2}=3, n_{3}=10, n_{4}=2, n_{5}=0, n_{6}=1
$$

this graph is nonconnected, then this subcase is impossible.

Subcase 4:

$$
n_{4}=0, n_{5}=1, n_{6}=1, n_{1}+n_{2}=14, n_{3}=n_{1}+1
$$

The 5 and 6-vertices are adjacent only to 3 -vertices and therefore the number of 3 -vertices is greater than or equal to 11 . Because of $n_{2} \geq 3$ we now have $n_{1}=n_{3}-1 \geq 10$. Then, in this subcase we get

Table 1. Cases of $n_{4}=0, n_{5}=1, n_{6}=1, n_{1}+n_{2}=14, n_{3}=n_{1}+1$.

\begin{tabular}{lllllll}
\hline & $\boldsymbol{n}_{1}$ & $\boldsymbol{n}_{2}$ & $\boldsymbol{n}_{3}$ & $\boldsymbol{n}_{4}$ & $\boldsymbol{n}_{5}$ & $\boldsymbol{n}_{6}$ \\
\hline (a) & 10 & 4 & 11 & 0 & 1 & 1 \\
(b) & 11 & 3 & 12 & 0 & 1 & 1 \\
\hline
\end{tabular}

the only case (a) can occurs and its graph is as follows.

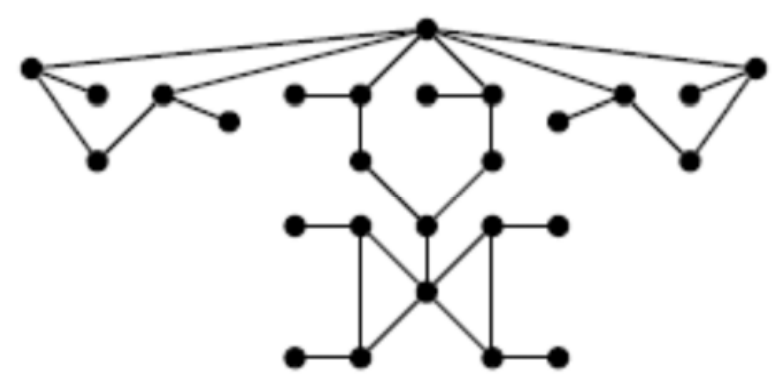

Figure 1. First member of a family of 3-harmonic graphs which $c=5$ and $\Delta=6$.

Subcase 5:

$$
n_{4}=1, n_{5}=1, n_{6}=1, n_{1}+n_{2}=16, n_{3}=n_{1}-1
$$

Since 3-vertices adjacent to 4-vertex, 5-vertex, 6-vertex, are distinct, so we need at least 15,3-vertices, then $n_{1} \geq 16$. It result that $n_{1}+n_{2} \geq 19$ that impossible.

Subcase 6:

$$
n_{4}=2, n_{5}=1, n_{6}=1, n_{1}+n_{2}=18, n_{3}=n_{1}-3
$$

Similar arguments Subcase 1.5 this subcase is impossible. Subcase 7:

$$
n_{4}=0, n_{5}=0, n_{6}=2, n_{1}+n_{2}=16, n_{3}=n_{1}
$$

Since 3 -vertices adjacent to 6-vertices are distinct, so we need at least 12,3 -vertices and 6, 2-vertices, then $n_{1} \geq 12$. It result that $n_{1}+n_{2}=n_{3}+n_{2} \geq 18$ that impossible.

Subcase 8:

$$
n_{4}=1, n_{5}=0, n_{6}=2, n_{1}+n_{2}=20, n_{3}=n_{1}-2
$$

In this subcase we have $n_{3} \geq 16$ then $n_{1} \geq 18$ and $n_{1}+n_{2} \geq 21$ that impossible.

Subcase 9:

$$
n_{4}=2, n_{5}=0, n_{6}=2, n_{1}+n_{2}=22, n_{3}=n_{1}-4
$$

In this subcase we have $n_{3} \geq 16$ then $n_{1} \geq 20$ and $n_{1}+n_{2} \geq 23$ that impossible.

Subcase 10:

$$
n_{4}=0, n_{5}=1, n_{6}=2, n_{1}+n_{2}=23, n_{3}=n_{1}-3
$$

In this subcase we have $n_{3} \geq 17$ therefore $n_{1} \geq 20$ and $n_{1}+n_{2} \geq 26$ that this is a contradiction.

Subcase 11:

$$
n_{4}=1, n_{5}=1, n_{6}=2, n_{1}+n_{2}=25, n_{3}=n_{1}-5
$$

In this subcase we have $n_{3} \geq 21$ thus $n_{1} \geq 26$ and $n_{1}+n_{2} \geq 32$ that this cannot happen.

Subcase 12:

$$
n_{4}=2, n_{5}=1, n_{6}=2, n_{1}+n_{2}=27, n_{3}=n_{1}-7
$$

In this subcase we get $n_{3} \geq 21$ hence $n_{1} \geq 28$ and $n_{1}+n_{2} \geq 34$ that impossible.

\section{Case 2: $\lambda=3, \Delta=5$}

Lemma 5 follows that $n_{3}-n_{1} \geq 0$. Equations (6) and (7) now became

$$
-2 n_{1}-2 n_{2}+4 n_{4}+10 n_{5}+4 n_{6}=0
$$

and

$$
-n_{1}+n_{3}+2 n_{4}+3 n_{5}=8 .
$$

We have following subcases:

Subcase 13:

$$
n_{4}=0, n_{5}=2, n_{1}+n_{2}=10, n_{3}=n_{1}+2
$$

By the Lemma 7, every 5-vertex is adjacent only with 3- 
vertices. Therefore $n_{3} \geq 10$ and $n_{1} \geq 8$, then we have

Table 2. Cases of $n_{4}=0, n_{5}=2, n_{1}+n_{2}=10, n_{3}=n_{1}+2$.

\begin{tabular}{llllll}
\hline & $\boldsymbol{n}_{1}$ & $\boldsymbol{n}_{2}$ & $\boldsymbol{n}_{3}$ & $\boldsymbol{n}_{4}$ & $\boldsymbol{n}_{5}$ \\
\hline (a) & 8 & 2 & 10 & 0 & 2 \\
(b) & 9 & 1 & 11 & 0 & 2 \\
(c) & 10 & 0 & 12 & 0 & 2 \\
\hline
\end{tabular}

Case (b) does not hold, but for case (a) we have 3 harmonic graphs as follow:
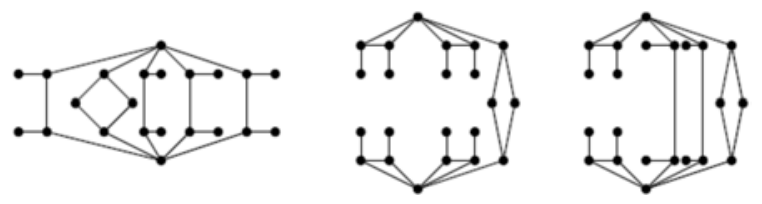

Figure 2. First members of a family of 3-harmonic graphs which $c=5$ and $\Delta=5$.

and for case (c) we have 8 harmonic graphs as follow:
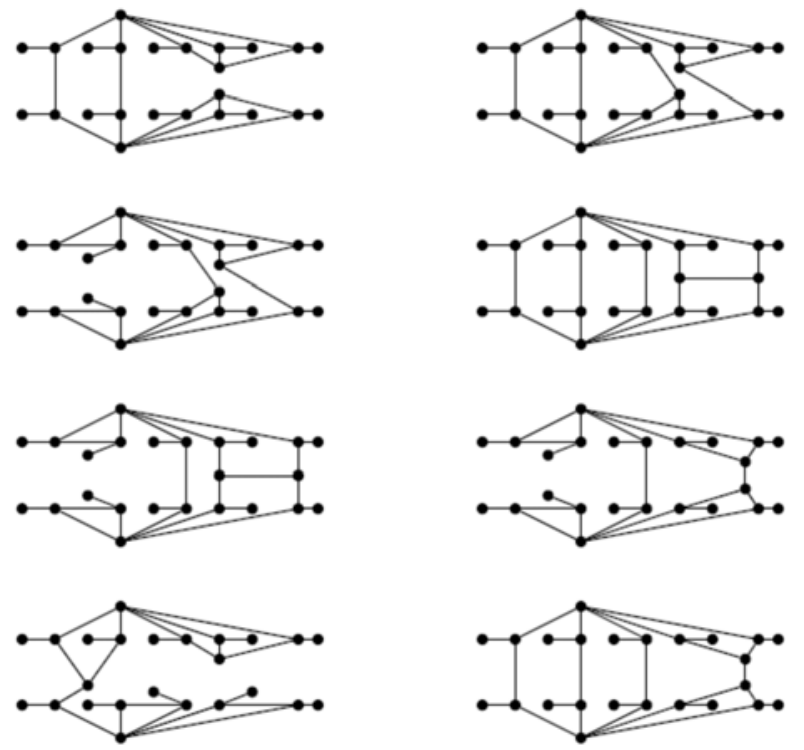

Figure 3. Second members of a family of 3-harmonic graphs which $c=5$ and $\Delta=5$.

Subcase 14:

$$
n_{4}=1, n_{5}=2, n_{1}+n_{2}=12, n_{3}=n_{1}
$$

In this subcase we have $n_{3} \geq 14$ then $n_{1} \geq 14$ and $n_{1}+n_{2} \geq 14$ that impossible.

Subcase 15:

$$
n_{4}=2, n_{5}=2, n_{1}+n_{2}=14, n_{3}=n_{1}-2
$$

Since $n_{3}-n_{1} \geq 0$ then this subcase is impossible.

Subcase 16:

$$
n_{4}=0, n_{5}=1, n_{1}+n_{2}=5, n_{3}=n_{1}+5
$$

In this subcase we have $n_{3} \geq 5$ then we have
Table 3. Cases of $n_{4}=0, n_{5}=1, n_{1}+n_{2}=5, n_{3}=n_{1}+5$.

\begin{tabular}{llllll}
\hline & $\boldsymbol{n}_{1}$ & $\boldsymbol{n}_{2}$ & $\boldsymbol{n}_{3}$ & $\boldsymbol{n}_{4}$ & $\boldsymbol{n}_{5}$ \\
\hline (a) & 0 & 5 & 5 & 0 & 1 \\
(b) & 1 & 4 & 6 & 0 & 1 \\
(c) & 2 & 3 & 7 & 0 & 1 \\
(d) & 3 & 2 & 8 & 0 & 1 \\
(e) & 4 & 1 & 9 & 0 & 1 \\
(f) & 5 & 0 & 10 & 0 & 1 \\
\hline
\end{tabular}

cases (b), (c) and (e) do not hold, but for case (a) we have 2 harmonic graphs as follow:
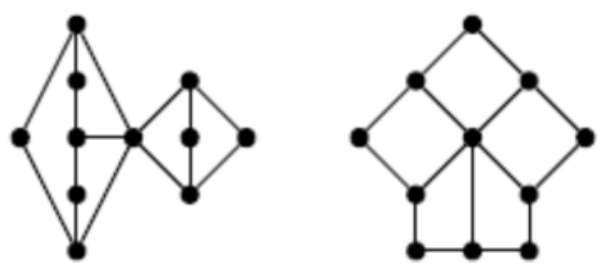

Figure 4. Third members of a family of 3-harmonic graphs which $c=5$ and $\Delta=5$.

for case (d) we have 1 harmonic graphs as follow:

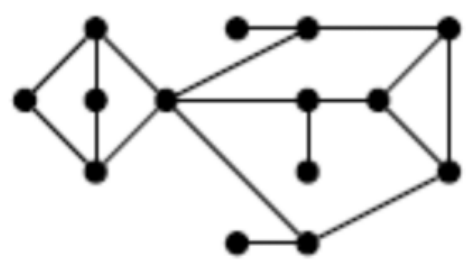

Figure 5. Fourth members of a family of 3-harmonic graphs which $c=5$ and $\Delta=5$.

for case (f) we have 3 harmonic graphs as follow:
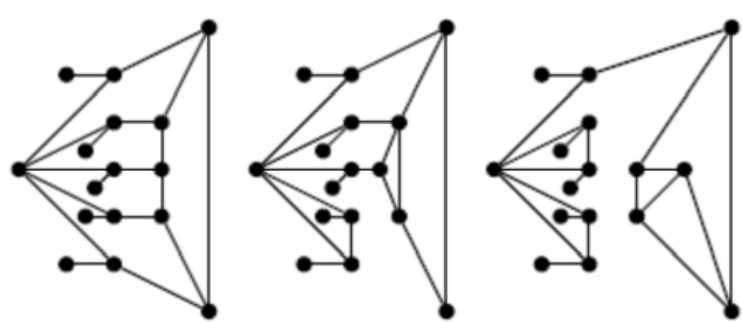

Figure 6. Fifth members of a family of 3-harmonic graphs which $c=5$ and $\Delta=5$.

Subcase 17:

$$
n_{4}=1, n_{5}=1, n_{1}+n_{2}=7, n_{3}=n_{1}+3
$$

In this subcase we have $n_{3} \geq 9$ then $n_{1} \geq 6$ and

$$
\text { Table 4. Cases of } n_{4}=1, n_{5}=1, n_{1}+n_{2}=7, n_{3}=n_{1}+3 \text {. }
$$

\begin{tabular}{llllll}
\hline & $\boldsymbol{n}_{1}$ & $\boldsymbol{n}_{2}$ & $\boldsymbol{n}_{3}$ & $\boldsymbol{n}_{4}$ & $\boldsymbol{n}_{5}$ \\
\hline (a) & 6 & 1 & 9 & 1 & 1 \\
(b) & 7 & 0 & 10 & 1 & 1 \\
\hline
\end{tabular}


since the neighbors 3 -vertex adjacent to 4-vertex has a 2vertex, a 3-vertex, so we need at least 2, 2-vertices, then this subcase is impossible.

Subcase 18:

$$
n_{4}=2, n_{5}=1, n_{1}+n_{2}=9, n_{3}=n_{1}+1
$$

In this subcase we have $n_{3} \geq 9$ hence $n_{1} \geq 8$ and

Table 5. Cases of $n_{4}=2, n_{5}=1, n_{1}+n_{2}=9, n_{3}=n_{1}+1$.

\begin{tabular}{llllll}
\hline & $\boldsymbol{n}_{1}$ & $\boldsymbol{n}_{2}$ & $\boldsymbol{n}_{3}$ & $\boldsymbol{n}_{4}$ & $\boldsymbol{n}_{5}$ \\
\hline (a) & 8 & 1 & 9 & 2 & 1 \\
(b) & 9 & 0 & 10 & 2 & 1 \\
\hline
\end{tabular}

since some the neighbors 3 -vertex adjacent to 5 -vertex has a 2-vertex, a 3-vertex, so we need at least 2, 2-vertices, then this subcase is impossible.

Case 3: $\lambda=3, \Delta=4$

Lemma 5 follows that $n_{3}-n_{1} \geq 0$. Equations (6) and (7) now became

$$
-2 n_{1}-2 n_{2}+4 n_{4}=0
$$

and

$$
-n_{1}+n_{3}+2 n_{4}=8
$$

$n_{3}-n_{1} \geq 0$ results that $1 \leq n_{4} \leq 4$ and the other hand $n_{1}+n_{2}=2 n_{4}$, then we have following subcases:

Subcase 19:

$$
n_{4}=1, n_{1}+n_{2}=2, n_{3}=n_{1}+6
$$

Equivalently

Table 6. Cases of $n_{4}=1, n_{1}+n_{2}=2, n_{3}=n_{1}+6$.

\begin{tabular}{lllll}
\hline & $\boldsymbol{n}_{1}$ & $\boldsymbol{n}_{2}$ & $\boldsymbol{n}_{3}$ & $\boldsymbol{n}_{4}$ \\
\hline (a) & 1 & 1 & 7 & 1 \\
(b) & 2 & 0 & 8 & 1 \\
(c) & 0 & 2 & 6 & 1 \\
\hline
\end{tabular}

since the neighbors 3-vertex adjacent to 4-vertex has a 2vertex, a 3-vertex, so we need at least 2, 2-vertices, then subcases (a) and (b) are impossible, but for case (c) we have 2 harmonic graphs as follow:
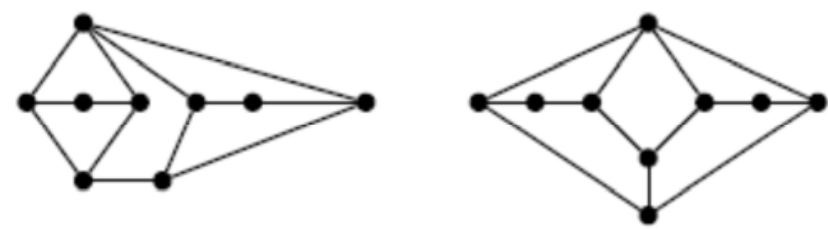

Figure7. First members of a family of 3-harmonic graphs which $c=5$ and $\Delta=4$.
Subcase 20:

$$
n_{4}=2, n_{1}+n_{2}=4, n_{3}=n_{1}+4
$$

equivalently

Table 7. Cases of $n_{4}=2, n_{1}+n_{2}=4, n_{3}=n_{1}+4$

\begin{tabular}{lllll}
\hline & $\boldsymbol{n}_{1}$ & $\boldsymbol{n}_{2}$ & $\boldsymbol{n}_{3}$ & $\boldsymbol{n}_{4}$ \\
\hline (a) & 0 & 4 & 4 & 2 \\
(b) & 1 & 3 & 5 & 2 \\
(c) & 2 & 2 & 6 & 2 \\
(d) & 3 & 1 & 7 & 2 \\
(e) & 4 & 0 & 8 & 2 \\
\hline
\end{tabular}

for case (a) we have 3 harmonic graphs as follow:
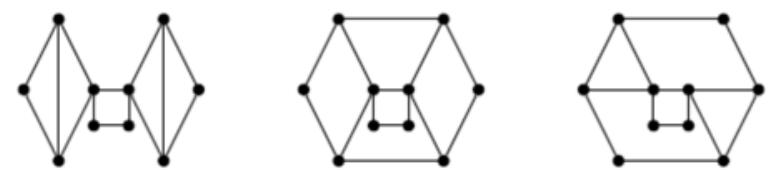

Figure 8. Second members of a family of 3-harmonic graphs which $c=5$ and $\Delta=4$.

In subcase (b) we need at least 6, 3-vertices, then this subcase is impossible, also subcases (d) and (e) are impossible, but for case (c) we have 3 harmonic graphs as follow:

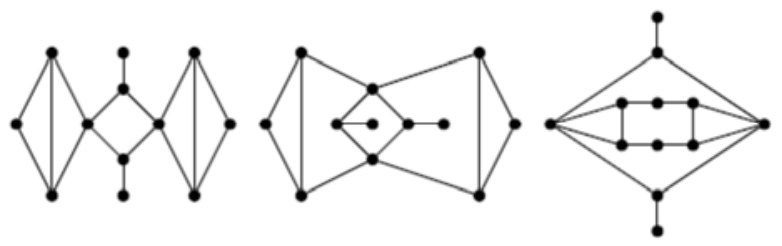

Figure 9. Third members of a family of 3-harmonic graphs which $c=5$ and $\Delta=4$.

\section{Subcase 21:}

$$
n_{4}=3, n_{1}+n_{2}=6, n_{3}=n_{1}+2
$$

If any two 4-vertices of three 4-vertices be adjacent, then $n_{3}=0$, thus this manner cannot occurs. If just a 4-vertex adjacent with the other 4 -vertices, then we have 2 harmonic graphs as follow:
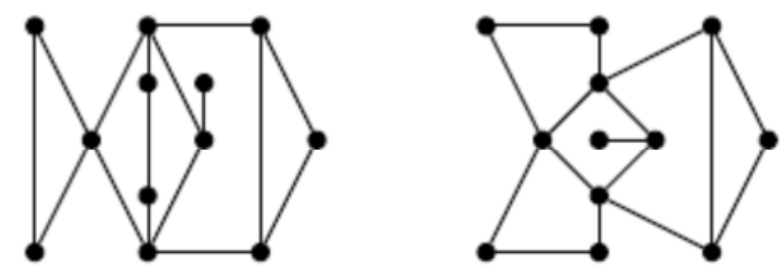

Figure 10. Fourth members of a family of 3-harmonic graphs which $c=5$ and $\Delta=4$

if the only two 4-vertices of three 4-vertices be adjacent, then we have 2 harmonic graphs as follow: 

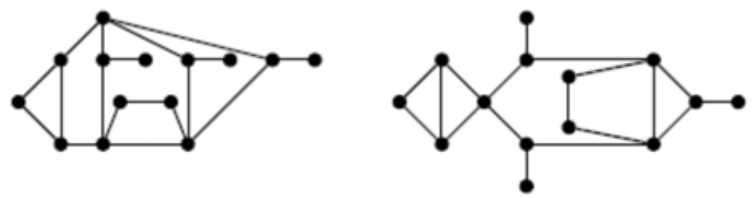

Figure 11. Fifth members of a family of 3-harmonic graphs which $c=5$ and $\Delta=4$.

Subcase 22:

$$
n_{4}=4, n_{1}+n_{2}=8, n_{3}=n_{1}
$$

equivalently

Table 8. Cases of $n_{4}=4, n_{1}+n_{2}=8, n_{3}=n_{1}$.

\begin{tabular}{lllll}
\hline & $\boldsymbol{n}_{1}$ & $\boldsymbol{n}_{2}$ & $\boldsymbol{n}_{3}$ & $\boldsymbol{n}_{4}$ \\
\hline (a) & 0 & 8 & 0 & 4 \\
(b) & 1 & 7 & 1 & 4 \\
(c) & 2 & 6 & 2 & 4 \\
(d) & 3 & 5 & 3 & 4 \\
(e) & 4 & 4 & 4 & 4 \\
(f) & 5 & 3 & 5 & 4 \\
(g) & 6 & 2 & 6 & 4 \\
(h) & 7 & 1 & 7 & 4 \\
(k) & 8 & 0 & 8 & 4 \\
\hline
\end{tabular}

In above subcases we need to the even number of 3vertices, then these subcases (b), (d), (e) and (h) are impossible. For case (a) we have 4 harmonic graphs as follow:
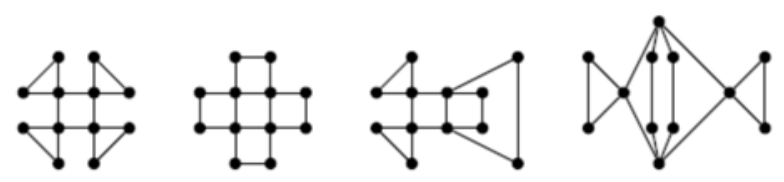

Figure 12. Sixth members of a family of 3-harmonic graphs which $c=5$ and $\Delta=4$.

for case (c) we have 3 harmonic graphs as follow:
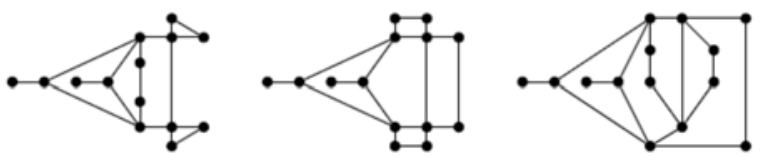

Figure 13. Seventh members of a family of 3-harmonic graphs which $c=5$ and $\Delta=4$.

for case (e) we have 2 harmonic graphs as follow:
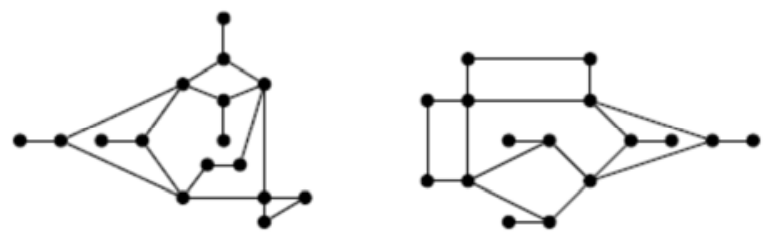

Figure 14. Eighth members of a family of 3-harmonic graphs which $c=5$ and $\Delta=4$.

for case (g) we have 2 harmonic graphs as follow:
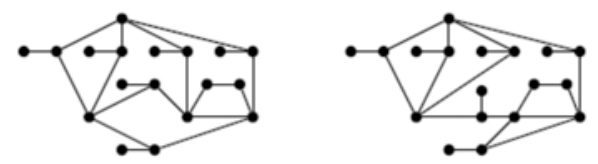

Figure 15. Ninth members of a family of 3-harmonic graphs which $c=5$ and $\Delta=4$.

and also for case (k) we have 3 harmonic graphs as follow:

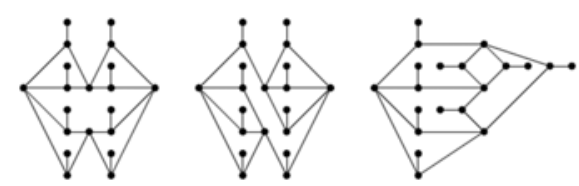

Figure 16. Tenth members of a family of 3-harmonic graphs which $c=5$ and $\Delta=4$.

\section{Case 4: $\lambda=4, \Delta=12$}

Lemma 5 follows that $2 n_{4}-n_{1} \geq 0$. Equations (6) and (7) now became

$$
\begin{aligned}
& -3 n_{1}-4 n_{2}-3 n_{3}+5 n_{5}+12 n_{6}+21 n_{7}+32 n_{8} \\
& +45 n_{9}+60 n_{10}+77 n_{11}+96 n_{12}=0,
\end{aligned}
$$

and

$$
\begin{aligned}
& -n_{1}+n_{3}+2 n_{4}+3 n_{5}+4 n_{6}+5 n_{7}+6 n_{8} \\
& +7 n_{9}+8 n_{10}+9 n_{11}+10 n_{12}=8 .
\end{aligned}
$$

Since $n_{12} \geq 1$ and $2 n_{4}-n_{1} \geq 0$ then this case is impossible. Case 5: $\lambda=4, \Delta=11$

Lemma 5 follows that $2 n_{4}-n_{1} \geq 0$. Equations (6) and (7) now became

$$
\begin{aligned}
& -3 n_{1}-4 n_{2}-3 n_{3}+5 n_{5}+12 n_{6}+21 n_{7} \\
& +32 n_{8}+45 n_{9}+60 n_{10}+77 n_{11}=0,
\end{aligned}
$$

and

$$
\begin{aligned}
& -n_{1}+n_{3}+2 n_{4}+3 n_{5}+4 n_{6}+5 n_{7} \\
& +6 n_{8}+7 n_{9}+8 n_{10}+9 n_{11}=8 .
\end{aligned}
$$

Because of $n_{11} \geq 1$ and $2 n_{4}-n_{1} \geq 0$ this cannot happen.

Case 6: $\lambda=\mathbf{4}, \Delta=\mathbf{1 0}$

Lemma 5 follows that $2 n_{4}-n_{1} \geq 0$. Equations (6) and (7) now became

$$
\begin{aligned}
& -3 n_{1}-4 n_{2}-3 n_{3}+5 n_{5}+12 n_{6} \\
& +21 n_{7}+32 n_{8}+45 n_{9}+60 n_{10}=0
\end{aligned}
$$

and

$$
\begin{aligned}
& -n_{1}+n_{3}+2 n_{4}+3 n_{5}+4 n_{6} \\
& +5 n_{7}+6 n_{8}+7 n_{9}+8 n_{10}=8 .
\end{aligned}
$$

Since $n_{10} \geq 1$ and $2 n_{4}-n_{1} \geq 0$ then (45) implies that 


$$
\begin{aligned}
& n_{10}=1,2 n_{4}=n_{1}, \\
& n_{3}=n_{5}=n_{6}=n_{7}=n_{8}=n_{9}=0
\end{aligned}
$$

and (45) results that $3 n_{1}+4 n_{2}=60$. Since vertices adjacent to 10 -vertex are 4 -vertex then $n_{4} \geq 10$ and $n_{1} \geq 20$. The equation $3 n_{1}+4 n_{2}=60$ leads to $n_{1}=20, n_{2}=0, n_{4}=10$. This harmonic graph as follow:

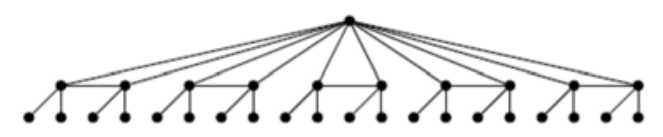

Figure 17. First member of a family of 4-harmonic graphs which $c=5$ and $\Delta=10$.

Case 7: $\lambda=4, \Delta=9$

From Lemma 5 we get $2 n_{4}-n_{1} \geq 0$. Equations (6) and (7) imply that

$$
\begin{aligned}
& -3 n_{1}-4 n_{2}-3 n_{3}+5 n_{5}+12 n_{6} \\
& +21 n_{7}+32 n_{8}+45 n_{9}=0
\end{aligned}
$$

and

$$
\begin{aligned}
& -n_{1}+n_{3}+2 n_{4}+3 n_{5}+ \\
& 4 n_{6}+5 n_{7}+6 n_{8}+7 n_{9}=8 .
\end{aligned}
$$

Because of $n_{9} \geq 1$ and $2 n_{4}-n_{1} \geq 0$ the relation (48) implies that

$$
n_{9}=1,2 n_{4}=n_{1}, n_{3}=1, n_{5}=n_{6}=n_{7}=n_{8}=0
$$

or

$$
n_{9}=1,2 n_{4}-1=n_{1}, n_{3}=n_{5}=n_{6}=n_{7}=n_{8}=0
$$

and (47) results that $3 n_{1}+4 n_{2}+3 n_{3}=45$. Since vertices adjacent to 9-vertex are 4 -vertex then $n_{4} \geq 9$ and $n_{1} \geq 17$. The equation $3 n_{1}+4 n_{2}+3 n_{3}=45$ results that this case is impossible.

Case 8: $\lambda=4, \Delta=8$

Lemma 5 leads to $2 n_{4}-n_{1} \geq 0$. From equations (6) and (7) we get

$$
\begin{aligned}
& -3 n_{1}-4 n_{2}-3 n_{3}+5 n_{5} \\
& +12 n_{6}+21 n_{7}+32 n_{8}=0
\end{aligned}
$$

and

$$
-n_{1}+n_{3}+2 n_{4}+3 n_{5}+4 n_{6}+5 n_{7}+6 n_{8}=8 .
$$

Since $n_{8} \geq 1$ and $2 n_{4}-n_{1} \geq 0$ then (52) implies that

$$
n_{8}=1,2 n_{4}-n_{1}+n_{3}=2, n_{5}=n_{6}=n_{7}=0
$$

and (51) implies that $3 n_{1}+4 n_{2}+3 n_{3}=32$. Since vertices adjacent to 8 -vertex are 4 -vertex so $n_{4} \geq 8$ and $n_{1} \geq 14$. The equation $3 n_{1}+4 n_{2}+3 n_{3}=32$ results that this case is impossible.

Case 9: $\lambda=4, \Delta=7$

From Lemma 5 we have $2 n_{4}-n_{1} \geq 0$. Equations (6) and (7) lead to

$$
-3 n_{1}-4 n_{2}-3 n_{3}+5 n_{5}+12 n_{6}+21 n_{7}=0,
$$

and

$$
-n_{1}+n_{3}+2 n_{4}+3 n_{5}+4 n_{6}+5 n_{7}=8
$$

Since $n_{7} \geq 1$ and $2 n_{4}-n_{1} \geq 0$ then (55) implies that

$$
n_{7}=1,2 n_{4}-n_{1}+n_{3}+3 n_{5}=3, n_{6}=0
$$

and (54) results that $3 n_{1}+4 n_{2}+3 n_{3}-5 n_{5}=21$. If $n_{5}=0$ then vertices adjacent to 7 -vertex are 4 -vertex then $n_{4} \geq 7$ and $n_{1} \geq 11$. The equation $3 n_{1}+4 n_{2}+3 n_{3}=21$ results that this case is impossible. Also, if $n_{5}=1$ then $n_{3}=0$ and $2 n_{4}=n_{1}$, then since some of vertices adjacent to 7-vertex are 4 -vertex then $n_{4} \geq 5$ and $n_{1} \geq 10$. The equation $3 n_{1}+4 n_{2}=26$ results that this case is impossible.

Case 10: $\lambda=4, \Delta=6$

Lemma 5 follows that $2 n_{4}-n_{1} \geq 0$. Equations (6) and (7) now became

$$
-3 n_{1}-4 n_{2}-3 n_{3}+5 n_{5}+12 n_{6}=0,
$$

and

$$
-n_{1}+n_{3}+2 n_{4}+3 n_{5}+4 n_{6}=8
$$

We have following subcases:

Subcase 23:

$$
\begin{aligned}
& n_{6}=1,2 n_{4}=n_{1}, n_{3}+3 n_{5}=4, \\
& -3 n_{1}-4 n_{2}-3 n_{3}+5 n_{5}+12=0
\end{aligned}
$$

If $n_{5}=0$ then $n_{3}=4, n_{1}=n_{2}=n_{4}=0$ therefore this manner not occurs. If $n_{5}=1$ then $n_{3}=1$ and $3 n_{1}+4 n_{2}=14$. On the other hand $n_{4} \geq 4$ thus $n_{1} \geq 8$ that this contradiction with $3 n_{1}+4 n_{2}=14$. Hence this manner also cannot occurs.

Subcase 24:

$$
\begin{gathered}
n_{6}=1,2 n_{4}=n_{1}+1, n_{3}+3 n_{5}=3 \\
-3 n_{1}-4 n_{2}-3 n_{3}+5 n_{5}+12=0
\end{gathered}
$$

If $n_{5}=0$ then $n_{3}=3, n_{1}=1, n_{2}=0, n_{4}=1$ therefore this manner is impossible. If $n_{5}=1$ then $n_{3}=0$ and $3 n_{1}+4 n_{2}=17$. Also, $n_{4} \geq 4$ thus $n_{1} \geq 8$ that this contradiction with $3 n_{1}+4 n_{2}=17$. Therefore this case also cannot occurs. 
Subcase 25:

$$
\begin{aligned}
& n_{6}=1,2 n_{4}=n_{1}+2, n_{3}+3 n_{5}=2, \\
& -3 n_{1}-4 n_{2}-3 n_{3}+5 n_{5}+12=0
\end{aligned}
$$

In this subcases $n_{3}=2, n_{5}=0, n_{1}=2, n_{2}=0, n_{4}=2$ then this case is impossible.

Subcase 26.

$$
\begin{gathered}
n_{6}=1,2 n_{4}=n_{1}+3, n_{3}+3 n_{5}=1, \\
-3 n_{1}-4 n_{2}-3 n_{3}+5 n_{5}+12=0
\end{gathered}
$$

In this subcases $n_{3}=1, n_{5}=0, n_{1}=3, n_{2}=0, n_{4}=3$ then this case cannot happen.

Subcase 27.

$$
\begin{aligned}
& n_{6}=1,2 n_{4}=n_{1}+4, n_{3}+3 n_{5}=0, \\
& -3 n_{1}-4 n_{2}-3 n_{3}+5 n_{5}+12=0
\end{aligned}
$$

In this subcases $n_{3}=n_{5}=0,3 n_{1}+4 n_{2}=12$. Since vertices adjacent to 6 -vertex are 4 -vertex then $n_{4} \geq 6$ and $n_{1} \geq 8$. So this contradiction with $3 n_{1}+4 n_{2}=12$. Then this case is impossible.

Subcase 28:

$$
\begin{aligned}
& n_{6}=2,2 n_{4}=n_{1}, n_{3}=n_{5}=0 \\
& 3 n_{1}+4 n_{2}=24
\end{aligned}
$$

since some vertices adjacent to 6-vertex are 4-vertex then $n_{4} \geq 4$ and $n_{1} \geq 8, n_{2} \geq 2$. That this contradiction with $3 n_{1}+4 n_{2}=24$, then this case is impossible.

Case 11: $\lambda=4, \Delta=5$

Lemma 5 follows that $2 n_{4}-n_{1} \geq 0$. Equations (6) and (7) now became

$$
-3 n_{1}-4 n_{2}-3 n_{3}+5 n_{5}=0
$$

and

$$
-n_{1}+n_{3}+2 n_{4}+3 n_{5}=8 \text {. }
$$

$3 n_{1}+4 n_{2}=5 n_{5}-3 n_{3} \geq 0$ then we have following subcases:

Subcase 29:

$$
n_{5}=1, n_{3}=0,2 n_{4}=n_{1}+5,3 n_{1}+4 n_{2}=5
$$

Since vertices adjacent to 5-vertex are 4-vertex then $n_{4}=5$ and $n_{1}=5$ that this contradiction with $3 n_{1}+4 n_{2}=5$. Hence this case is impossible.

Subcase 30:

$$
n_{5}=1, n_{3}=1,2 n_{4}=n_{1}+4,3 n_{1}+4 n_{2}=2
$$

Since $n_{1} \geq 0$ and $n_{2} \geq 0$, hence this case cannot happen. Subcase 31:

$$
n_{5}=2, n_{3}=0,2 n_{4}=n_{1}+2,3 n_{1}+4 n_{2}=10
$$

Since some vertices adjacent to 5-vertex are 4-vertex then $n_{4} \geq 3$ and $n_{1} \geq 4$ that this contradiction with $3 n_{1}+4 n_{2}=10$. Therefore this case is impossible.

Subcase 32:

$$
n_{5}=2, n_{3}=1,2 n_{4}=n_{1}+1,3 n_{1}+4 n_{2}=7
$$

Some vertices adjacent to 5-vertex are 4-vertex then $n_{4} \geq 3$ and $n_{1} \geq 5$ that this contradiction with $3 n_{1}+4 n_{2}=7$. Thus this case is impossible.

Subcase 33:

$$
n_{5}=2, n_{3}=2,2 n_{4}=n_{1}, 3 n_{1}+4 n_{2}=4
$$

Since some vertices adjacent to 5 -vertex are 4-vertex then $n_{4} \geq 3$ and $n_{1} \geq 6$ that this contradiction with $3 n_{1}+4 n_{2}=4$. Hence this case is impossible.

\section{Definition 3:}

A graph is planar if it can be drawn in a plane without graph edges crossing.

\section{Corollary 1:}

All of c-cyclic nonregular harmonic graphs for $c \leq 5$ are planar graphs.

\section{Regular Harmonic Graphs}

If a pentacyclic $\lambda$-harmonic graph be regular then we have $n=\frac{8}{\lambda-2}$ and $n \geq \lambda+1$, therefore we have the only $\lambda=3, n=8$. In this case we have 4, 3-harmonic graphs as follow:

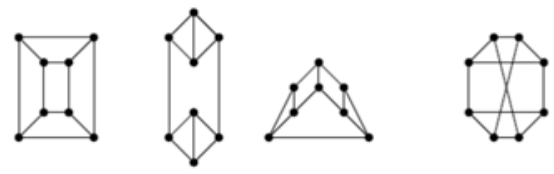

Figure 18. Connected regular pentacyclic harmonic graphs.

\section{Conclusions}

Let \# r(c) and \#nr(c) be denote the number of connected ccyclic regular and nonregular harmonic graphs, respectively, for a fixed value c. According to fined results, the number of harmonic graphs as follows.

Table 9. The number of harmonic graphs.

\begin{tabular}{llll}
\hline $\mathbf{c}$ & $\# \mathbf{r}(\mathbf{c})$ & \#nr(c) & Remark \\
\hline 0 & 1 & $\infty$ & $\lambda \geq 1$ \\
1 & $\infty$ & 0 & $\lambda=2$ \\
2 & 0 & 0 & \\
3 & 1 & 4 & $\lambda=3$ \\
4 & 2 & 18 & $\lambda=3$ \\
5 & 4 & 45 & $\lambda=3, \lambda=4$ \\
$\geq 6$ & finite & finite & \\
\hline
\end{tabular}




\section{References}

[1] Borovicanin B., Grunewald S., Gutman I., Petrovic M. 2003. Harmonic graphs with small number of cycles, Discrete Mathematics 265, 31-44.

[2] Cvetkovi'c D., Doob M., Sachs H. 1995. Spectra of Graphs: Theory and Applications (3rd ed.), Johann Ambrosius Barth, Heidelberg.

[3] Cvetkovi'c D., Rowlinson P., Simi'c S. 1997. Eigenspaces of Graphs, Cambridge University Press, Cambridge.

[4] Dress A., Gutman I. 2003. Asymptotic results regarding the number of walks in a graph, Appl. Math. Lett., 16(3), 389-393.

[5] Dress A., Gutman I. 2003. On the number of walks in a graph, Appl. Math. Lett. 16(5), 797-801.

[6] Favaron O., Mah'eo M., Sacl'e J.F., 1993. Some eigenvalue properties in graphs (conjectures of GraLti-II), Discrete Math. 111, 197-220.

[7] Godsil C., Royle G. 2001. Algebraic Graph Theory, SpringerVerlag, New York.

[8] Grunewald S. 2002. Harmonic tree, App. Math. Lett. 15, 1001-1004.

[9] Petrovi'c M., Borovi'canin B., Radosavljevi'c Z. 2006. The integral 3-harmonic graphs, Linear Algebra and its Applications 416, 298-312.

[10] Mahmoodi A. 2013. Some Results on Harmonic Graphs, Gen. Math. Notes, 19(1), 53-59.

[11] Salehi Zarrin Ghabaei A., Azami S. 2014. Some properties of harmonic graphs, Advances in Environmental Biology, 8(11), 597-605. 